\title{
Anergy and deletion in post-thymic tolerance
}

\author{
Atsuo Ochi, Kouichi Yuh, Kiyoshi Migita and Yojiro Kawabe
}

Jpn. J. Clin. Immun., 14 (5) : 503 503, 1991.

\begin{abstract}
Antigen-specific $T$ cell tolerance can be achieved by either deletion or functional inactivation (anergy) of antigen reactive clones. While clonal deletion represents the predominant mechanism mediating thymic tolerance, both anergy and deletion may contribute to the development of peripheral tolerance. At present, the basis for the use of deletion versus anergy to engender the tolerant state is unknown. To investigate the processes involved in expression of these phenomena, we have examined the temporal relationship of de novo protein synthesis to expression of tolerance in mice primed with Staphylococcus aureus enterotoxin B (SEB), a bacterial superantigen that can induce both antigen specific clonal anergy and clonal deletion among peripheral $\mathrm{T}$ cells. Concomitant administration of SEB and the protein synthesis inhibitor cycloheximide ( $\mathrm{CHX}$ ) abrogated induction of SEB-specific anergy, but did not interfere with programmed cell death (PCD) of SEB-reactive $\mathrm{T}$ cells. Although
\end{abstract}

PCD of SEB-reactive splenic cells was not altered by $\mathrm{CHX}$ in vitro, this treatment markedly reduced PCD in thymocytes. The results demonstrate that the de novo protein synthesis required for clonal inactivation and deletion associated with induction of antigen-specific tolerance in the periphery occurs at different time points in relation to the tolerizing stimulus. Deletion begins and ends within a given time-frame (between 2 days and 7 days) after SEB -priming, while clonal anergy develops earlier and is ongoing over a longer time course, appearing to last for at least two months. Thus although these two phenomena are both triggered by the same event, interaction of SEB with a specific $T$ cell antigen receptor, their subsequent development appears temporally distinct and independent. The factors that influence the evolution to either the inactivated state or to cell death and deletion remain to be determined. 